\title{
Actitudes y preferencias de estudiantes de traducción inglés-español frente a recursos gramaticales del español asociados a anglicismos de frecuencia*
}

\author{
Attitudes and preferences of English-Spanish translation students in relation to \\ Spanish grammatical features associated with frequency Anglicisms
}

\author{
Carlos I. Echeverría Arriagada \\ Universidad Chileno-Británica de Cultura, c/ Santa Lucía 124, Santiago, Santiago, Chile. \\ echeverria.arriagada@gmail.com
}

\begin{abstract}
Este artículo estudia las actitudes y las preferencias alternativas de estudiantes de traducción inglés-español chilenos de lengua nativa española frente a cuatro recursos gramaticales del español asociados a anglicismos de frecuencia, de los cuales se consideraron 15 variantes. Los resultados, registrados mediante la aplicación de un cuestionario de preguntas abiertas y cerradas, muestran, por un lado, que los informantes exhibieron actitudes marcadamente favorables hacia los recursos de interés y, por otro lado, que las preferencias alternativas de los informantes, las cuales se dividieron en 15 grupos y fueron coherentes con las actitudes registradas, corresponden a fórmulas de sustitución relativamente estables.
\end{abstract}

Palabras clave: gramática española, anglicismos de frecuencia, actitudes lingüísticas, preferencias alternativas, traducción inglés-español.

This article studies the attitudes and alternative preferences of Chilean native Spanish-speaking English-Spanish translation students in relation to four Spanish grammatical features associated with frequency Anglicisms, of which 15 different variants were considered. The results, which were recorded using a questionnaire including both closed and open questions, showed, on the one hand, that the informants had markedly favorable attitudes towards the features of interest and, on the other hand, that the alternative preferences of the informants, which divided into 15 different groups and were consistent with the observed attitudes, correspond to relatively stable substitution formulas.

Keywords: Spanish grammar, frequency Anglicisms, language attitudes, alternative preferences, English-Spanish translation.

Este artículo se basa en Echeverría 2015, investigación que contó con el financiamiento parcial de una Beca de Incentivo a la Investigación otorgada por el Vicedecanato de Investigación y Postgrado de la Facultad de Humanidades de la Universidad de Santiago de Chile. 


\section{INTRODUCCIÓN}

Los anglicismos de frecuencia, que corresponden a casos del fenómeno de contacto entre lenguas denominado interferencia de frecuencia, consisten en el sobreuso, por influencia del inglés, de recursos pertenecientes a la lengua local (Vázquez-Ayora 1977; Lorenzo 1980, 1991, 1996; Rodríguez 2000, 2002). Así pues, a diferencia de lo que sucede con la mayoría de los fenómenos lingüísticos considerados tradicionalmente como interferenciales, la anomalía que se produce en estos casos es solo cuantitativa; en otras palabras, los recursos de la lengua cuyo uso resulta en anglicismos de frecuencia no constituyen en sí mismos extranjerismos, sino que el extranjerismo está en cada caso solo en el sobreuso (Echeverría, 2016).

Ahora bien, aun cuando los recursos lingüísticos asociados a anglicismos de frecuencia, como se ha dicho, no son en sí mismos extranjerismos, se trata de recursos en cierto sentido problemáticos (podríamos decir estilísticamente problemáticos) en situaciones de contacto con el inglés, en especial en el contexto de la traducción desde dicho idioma, que parece ser donde más abunda este tipo de anglicismo. Por otro lado, no pocas veces lo problemático de dichos recursos parece interpretarse de una manera extrema, por lo que estos terminan recibiendo, sin justificación alguna, un tratamiento de distanciamiento o derechamente despreciativo en la literatura sobre traducción y en las aulas en que se enseña esta práctica interidiomática, como si se tratase de elementos en sí mismos extraños a la lengua local (Echeverría 2011, 2016). En consecuencia, a nuestro juicio, los recursos asociados a anglicismos de frecuencia, sobre todo desde el punto de vista pedagógico, merecen atención más allá de la sola descripción de su sobreuso por influencia del inglés.

Considerando lo anterior, en el presente estudio se busca explorar, por un lado, el contexto actitudinal subyacente al empleo de estos recursos por parte de los futuros traductores y, por otro lado, el acervo de herramientas de que estos disponen para no depender de ellos. Más precisamente, se propone aquí el objetivo de describir las actitudes, entendidas como tendencias psicológicas que se expresan como valoraciones en algún grado favorables o desfavorables (Cooper y Fishman 1974; Eagly y Chaiken 1993; López 1993; Moreno 2005; Garrett 2010), y las preferencias alternativas de un grupo de estudiantes de traducción inglés-español, de lengua nativa española, frente a recursos gramaticales del español asociados a anglicismos de frecuencia.

Por lo que respecta a la decisión de limitar la investigación a actitudes hacia recursos pertenecientes al nivel gramatical, esta se debió principalmente a que dicho nivel se ha visto en alguna medida descuidado en los estudios relativos al contacto entre lenguas (Vázquez-Ayora 1977; Klein-Andreu 1985; Thomason y Kaufman 1988; Ivir 1991).

\section{Metodología}

\subsection{Recursos gramaticales considerados}

Para la selección de los recursos asociados a anglicismos de frecuencia se tomó como punto de partida la tesis doctoral de Rodríguez (2000, 2002), quien estudió, en 
traducciones de documentos informáticos del inglés al español, diversos recursos de este último idioma que, en su mayoría, han sido asociados a anglicismos de frecuencia en la bibliografía especializada:
1) adjetivos prenominales
2) adverbios en -mente
3) gerundios
4) construcciones pasivas con ser + participio
5) repeticiones "innecesarias"
6) la perífrasis "poder + infinitivo"
7) la expresión estar disponible

Las razones para basarse en el trabajo de Rodríguez son tres: en primer lugar, dicha autora intenta limitarse al nivel gramatical, que es el que aquí interesa; en segundo lugar, su lista de recursos lingüísticos fue elaborada a partir de una amplia revisión bibliográfica ${ }^{1}$; en tercer lugar, se trata de una investigación empírica relativamente actual en la que se estudian textos pertenecientes a un mismo género discursivo y en la que constata que todos los recursos lingüísticos considerados, salvo uno, cuentan con una frecuencia de aparición mayor en las traducciones.

Aquí quedaron fuera de consideración, sin embargo, el gerundio, las repeticiones llamadas innecesarias y la expresión estar disponible. El gerundio, porque se trata del único recurso que, en la investigación de dicha autora, resultó no contar con una frecuencia de aparición por página mayor en los textos traducidos; las repeticiones léxicas "innecesarias", porque determinar cuándo una repetición lingüística es necesaria resulta problemático; y la expresión estar disponible, porque su adscripción al nivel gramatical es objetable².

De este modo, los recursos lingüísticos aquí considerados (en adelante, recursos de interés) fueron los cuatro siguientes:

1) adjetivos prenominales, que son adjetivos que, ubicándose antes del sustantivo que modifican, "actúan sobre la referencia o intensión sin que su aplicación afecte a la extensión del término modificado" (Demonte 1999: 192).

2) adverbios en -mente, que constituyen palabras que se componen de "una unidad léxica adjetival, que se neutraliza simultáneamente en las formas femenino y singular, y un elemento sufijal invariable, -mente" (García-Page 1991: 186) y que pueden actuar sobre verbos, adjetivos, adverbios u oraciones enteras (Domínguez 1970; Real Academia Española 1973; Real Academia Española y Asociación de Academias de la Lengua Española 2009).

3) construcciones pasivas con ser + participio, que son construcciones atributivas cuya

\footnotetext{
Entre los autores que considera Rodríguez para la elaboración de la lista de los recursos lingüísticos por ella estudiados están Vázquez-Ayora (1977), Pratt (1980), Calvo (1983), García Yebra (1984), Aguado (1990), Arauz (1992), Lorenzo (1980, 1991, 1996), Alcaraz y Martínez (1997), Lázaro (1997), López y Minett (1997) y Grijelmo (1997), por nombrar algunos.

2 Rodríguez parece tratar esta expresión como recurso gramatical debido a que se trata de una expresión pluriverbal, esto es, formada por más de una palabra. Sin embargo, cuando aquí hablamos de recursos gramaticales no hacemos referencia meramente a recursos cuyos elementos están combinados de acuerdo con la gramática de la lengua, sino a recursos que pertenecen en sí mismos al nivel gramatical. La expresión estar disponible, al ser un sintagma, pertenece al primer grupo, mas no al segundo, tal como sucede, p. ej., con perro (perr- + -o) y sacacorchos (saca- + -corchos), ambos signos sintagmáticos.
} 
constitución con la cópula y el participio de un verbo transitivo hace que el sujeto sea paciente en lugar de agente (Alarcos 1969, 1999; Gili Gaya 1980; Montes 2003; Real Academia Española 1973; Real Academia Española y Asociación de Academias de la Lengua Española 2009).

4) perífrasis "poder + infinitivo", que corresponde a una perífrasis modal que, por el verbo auxiliar con que se forma, expresa posibilidad (Real Academia Española 1973, Real Academia Española y Asociación de Academias de la Lengua Española 2009; Gili Gaya 1980).

Adicionalmente, tomando en cuenta la posibilidad de que las diversas manifestaciones que tienen los recursos de interés en la realidad del discurso den lugar a actitudes igualmente diversas, se decidió considerar distintas variantes de los recursos de interés. Cabe subrayar, de todos modos, que al hacer esto no se pretende realizar una clasificación exhaustiva de los recursos de interés, ni mucho menos, sino solo enfrentar a los informantes a una mayor diversidad lingüística al momento de registrar sus actitudes y preferencias alternativas.

Para los adjetivos prenominales seguimos principalmente a la Real Academia Española y la Asociación de Academias de la Lengua Española $(2009,2010)$ y a Demonte (1999). Las variantes consideradas para este recurso quedaron limitadas a las siguientes:

1) adjetivos prenominales epitéticos (o simplemente epítetos), que "destacan una propiedad inherente, prototípica o característica del sustantivo al que modifican" (Real Academia Española y Asociación de Academias de la Lengua Española 2009: 913) (p. ej., Ayer hubo un hermoso día).

2) adjetivos prenominales adverbiales, que indican "la manera como el concepto o intensión de un término se aplica a un determinado referente" (Demonte 1999: 139) (p. ej., El futuro candidato se reunirá con el partido esta semana).

3) adjetivos prenominales que se asimilan a los determinantes, cuantificadores o pronombres, los cuales "han adquirido propiedades sintácticas y semánticas propias de los cuantificadores [...], de los determinantes [...] o de los pronombres" (Real Academia Española y Asociación de Academias de la Lengua Española 2010: 249) (p. ej., Distinta situación era la que se vivía en los años setenta).

Para los adverbios en -mente seguimos a Domínguez (1970), quien ofrece una clasificación según la parte de la oración sobre la que actúa el adverbio, y distinguimos las siguientes variantes:

1) adverbios en -mente que actúan sobre verbos (p. ej., Fernando se volteó bruscamente al oír su voz).

2) adverbios en -mente que actúan sobre adjetivos (p. ej., Debe entregar un proyecto suficientemente riguroso para que lo consideremos).

3) adverbios en -mente que actúan sobre otros adverbios (p. ej., Esa posibilidad está absolutamente fuera de alcance).

4) adverbios en -mente que actúan sobre oraciones enteras (p. ej., Definitivamente se trata de una alternativa que hay que tener en cuenta).

En el caso de las construcciones pasivas con ser + participio nos basamos en el trabajo de Montes (2003). Este autor, recurriendo al concepto de prototipo, propone una 
clasificación cuatripartita de las construcciones pasivas según una escala de pasividad:

1) construcción pasiva con paciente y agente humanos individualizados.

2) construcción pasiva con paciente humano individualizado y agente humano genérico o indeterminado.

3) construcción pasiva con paciente humano genérico y agente genérico o indeterminado.

4) construcción pasiva con paciente inanimado.

No obstante, aunque aquí se tomó como punto de partida esta clasificación, se decidió realizar modificaciones importantes sobre ella; específicamente, se dejó de lado el criterio "humano/no humano" en relación con el agente y el paciente, criterio que Montes relaciona, mediante argumentos etimológicos, con la pasividad de las construcciones, y en cambio se consideró en cada caso si el agente y el paciente están individuados ${ }^{3}$ o no, con lo cual se logra una clasificación abarcadora y coherente. Así, las variantes consideradas para este recurso quedaron limitadas a las siguientes:

1) construcciones pasivas con agente y paciente individuados (p. ej., Pedro fue regañado por su madre).

2) construcciones pasivas con agente no individuado y paciente individuado (p. ej., $L a$ víctima estaba siendo observada por alguien).

3) construcciones pasivas con agente individuado y paciente no individuado (p. ej., Alguien había sido visto por el testigo en la escena del crimen).

4) construcciones pasivas con agente y paciente no individuados (p. ej., Alguien de nosotros podría estar siendo observado en este instante).

Por último, para la perífrasis "poder + infinitivo" consideramos el aspecto léxico del verbo en infinitivo, siguiendo la conocida clasificación aspectual cuatripartita propuesta por Vendler (1967) y adoptada, entre otros, por la Real Academia Española y la Asociación de Academias de la Lengua Española $(2009,2010)$, en la que se distinguen verbos que denotan actividades, realizaciones, consecuciones y estados. Por tanto, en el caso de este recurso distinguimos las siguientes variantes:

1) perífrasis "poder + infinitivo" con verbos que denotan actividades (p. ej., Me gustaría poder vender libros en la universidad).

2) perífrasis "poder + infinitivo" con verbos que denotan realizaciones (p. ej., Recién ahora la municipalidad pudo construir el puente).

3) perífrasis "poder + infinitivo" con verbos que denotan consecuciones (p. ej., Concéntrate y podrás encontrar las llaves).

4) perífrasis "poder + infinitivo" con verbos que denotan estados (p. ej., Con esfuerzo podré tener todo el dinero que quiero).

\subsection{El cuestionario}

Para la recolección de datos se estimó idóneo aplicar un cuestionario de preguntas cerradas (para las actitudes) y abiertas (para las preferencias alternativas). Para el registro de las actitudes se incluyeron tres oraciones por cada variante considerada

Por individuación se entiende aquí, de acuerdo con Coseriu (1982), el tipo de determinación nominal consistente en una selección ("discriminación 'real' y externa" [1982: 299]) de tipo definido. Según dicho lingüista, la individualización es solo un tipo especial de individuación (individuación de un singular). 
y se solicitó a los informantes que las evaluasen en términos de aceptabilidad, imaginando su utilización en instancias de uso cuidado del lenguaje, mediante una escala de diferencial semántico (Osgood, Suci y Tannenbaum 1975; Urbán 1980) de cinco puntos ( 1 y 2: actitud desfavorable; 3: ausencia de actitud; 4 y 5: actitud favorable $)^{4}$. Por otro lado, para el registro de las preferencias alternativas de los informantes, se solicitó a estos que, de considerar que existe uno o más modos de expresar mejor el contenido de las oraciones proporcionadas en el cuestionario, los ilustrasen por escrito, sin un máximo de oraciones posibles establecido.

Adicionalmente, previéndose respuestas de tendencia favorable debido a lo observado anteriormente en un muy modesto estudio piloto en el que se estudiaron las actitudes de una pequeña muestra de estudiantes de traducción inglés-español de dos instituciones privadas chilenas (Echeverría 2014), para evitar la presencia de un sesgo de aquiescencia - del cual se sospechó en el estudio piloto- se incluyeron en el cuestionario, a modo de filtro, varias oraciones con distintos recursos lingüísticos que constituyen contravenciones a algunos de los preceptos de corrección lingüística habituales, no comunes en la lengua culta (Bosque y Demonte 1999; Gómez Torrego 2003; Rabanales 2005; Real Academia Española 2005; Real Academia Española y Asociación de Academias de la Lengua Española 2009, 2010). Las contravenciones consideradas fueron tres, cada una con tres oraciones:

1) construcciones dequeístas (p. ej., Reconoce de que yo tenía razón).

2) duplicación de pronombre como proclítico y enclítico (p. ej., Dijo que se iba a irse temprano).

3) construcciones pleonásticas no enfáticas (p. ej., Salga afuera de inmediato).

De este modo, si en un cuestionario más de la mitad de estos recursos obtenía respuestas favorables, este se desestimaba automáticamente.

Por último, para equilibrar en alguna medida el contenido del cuestionario, pero sin hacerlo demasiado largo, se incluyeron en él cuatro oraciones consideradas no controvertidas.

Así pues, el cuestionario quedó constituido por un total de 58 oraciones.

\subsection{Informantes}

En un principio, la población de estudiantes de traducción considerada se limitó a alumnos de cuarto y quinto año del programa de Licenciatura en Lingüística Aplicada a la Traducción (Inglés-Japonés e Inglés-Portugués) de la Universidad de Santiago de Chile. Para conseguir la participación de informantes dentro de dicho programa, con la intención de que la muestra fuese lo más grande posible en las circunstancias dadas, se abrió una convocatoria para que los alumnos tuviesen la oportunidad de participar de manera voluntaria en el estudio a través de Internet.

\footnotetext{
Autores como Thurstone (1928), según el que describir una actitud con solo un índice numérico es análogo hacer lo mismo con una mesa de cocina, probablemente verían el método empleado como reduccionista; sin embargo, la decisión de hacer el cuestionario del modo descrito es coherente con la noción de actitud aquí adoptada. Por supuesto, hacer un estudio más multidimensional, considerando más criterios de evaluación, sería de valor científico; mas este estudio se limita a estudiar actitudes en cuanto a la aceptabilidad de los recursos de interés, lo cual hace lícita la medición de estas con índices numérico únicos.
} 
Ahora bien, debido a que la muestra que se consiguió no lograba superar la del estudio piloto, se abrió mediante un nuevo correo electrónico una segunda convocatoria para lograr un mayor número de informantes, en la que se invitó también a estudiantes de tercer año a participar, y se convino también una visita a uno de los cursos del programa. De este modo se logró conseguir un total de 30 cuestionarios respondidos, de los cuales dos fueron desestimados, uno por incompleción y otro por sospecha de sesgo de aquiescencia. Por tanto, el número de informantes efectivos, con cuestionarios respondidos de manera válida, fue 28 .

\section{Resultados}

\subsection{Actitudes}

Al analizar las actitudes registradas se observó una marcada tendencia hacia la favorabilidad, tendencia que se manifiesta también al considerarse cada recurso de interés por separado. En el Cuadro 1 se agrupan las respuestas de los informantes según su correspondencia con actitudes favorables (puntuaciones 5 y 6 ), con actitudes desfavorables (puntuaciones 1 y 2) o con una ausencia de actitud (puntuación 3), sin considerar aún cada variante por separado:

Cuadro 1. Distribución de las actitudes

\begin{tabular}{|c|c|c|c|}
\hline Recurso $\quad$ Categoría & $\begin{array}{c}\text { Actitudes } \\
\text { desfavorables }\end{array}$ & $\begin{array}{l}\text { Ausencia de } \\
\text { actitud }\end{array}$ & $\begin{array}{l}\text { Actitudes } \\
\text { favorables }\end{array}$ \\
\hline Adjetivos prenominales & $28(11,24 \%)$ & $39(15,66 \%)$ & $182(73,09 \%)$ \\
\hline Adverbios en -mente & $16(4,76 \%)$ & $25(7,44 \%)$ & $295(87,80 \%)$ \\
\hline Construcciones pasivas & $85(25,30 \%)$ & $50(14,88 \%)$ & $201(59,82 \%)$ \\
\hline Per. "poder + infinitivo" & $23(6,85 \%)$ & $50(14,88 \%)$ & $263(78,27 \%)$ \\
\hline
\end{tabular}

La media aritmética y la desviación típica de las puntuaciones asignadas a cada recurso se muestran en la Figura 1: 
Figura 1. Media aritmética y desviación típica de las puntuaciones asignadas a los recursos de interés

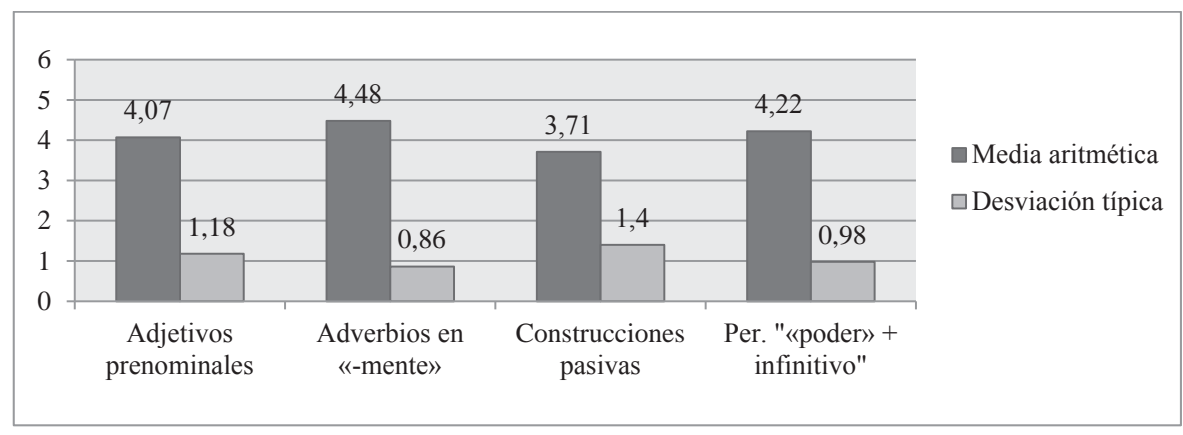

Se puede observar que todas las medias aritméticas se hallan en el segmento de la favorabilidad, siendo el recurso mejor puntuado los adverbios en -mente, seguidos de la perífrasis "poder + infinitivo", los adjetivos prenominales y por último las construcciones pasivas con ser + participio. Adicionalmente, las desviaciones típicas de las puntuaciones obtenidas para cada recurso también aquí resultaron ser relativamente bajas, lo que indica cierta homogeneidad en los resultados.

En cuanto a las distintas variantes de los recursos de interés, las medias aritméticas correspondientes a cada una de ellas se encuentran en su totalidad en el segmento de la favorabilidad. De un lado se destacan los adverbios en -mente, todas cuyas variantes obtuvieron medias superiores a 4, siendo la variante mejor puntuada los adverbios modificadores de otros adverbios, con una media de 4,64 (la más alta registrada en todo el cuestionario) y la peor puntuada los adverbios modificadores de oraciones, con una media de 4,38. Del otro lado se destacan las construcciones pasivas con ser + participio, que, además de tener solo una variante con una media superior a 4 (construcción con agente y paciente individuados, con 4,27), tuvo la variante peor puntuada del cuestionario (construcción con agente y paciente no individuados, con una media de 3,25). En cuanto a las desviaciones típicas, estas siguen siendo relativamente bajas, destacándose también a este respecto los adverbios en -mente, con todas sus desviaciones menores a 1 , y las construcciones pasivas con ser + participio, con todas sus desviaciones sobre 1 .

\subsection{Preferencias alternativas}

Cabe en este punto recordar que nuestro objetivo en esta sección es registrar las preferencias alternativas de los informantes, por lo cual no se tomó en cuenta ninguna respuesta en que no se sustituyesen los recursos de interés. Asimismo, no se consideraron aquí las respuestas en que, aun sustituyéndose el recurso de interés en cuestión, este se siguiese empleando de alguna otra forma. Tal fue el caso, p. ej., de Aquella vez actué de manera totalmente errónea (< Aquella vez hice las cosas completamente mal) ${ }^{5}$, donde se sustituyó todo el predicado, incluido el adverbio

Cada vez que se cite una oración de las proporcionadas alternativamente por los informantes, junto a ella irá, entre 
completamente, que es el recurso de interés, pero usándose totalmente, adverbio con la misma terminación.

Aplicado este nuevo filtro, se registró un total de 230 oraciones alternativas. La distribución de estas en relación con los distintos recursos de interés se muestra en el Cuadro 2 a continuación:

Cuadro 2. Distribución general de las oraciones alternativas registradas

\begin{tabular}{|l|c|}
\hline \multicolumn{1}{|c|}{ Recurso } & Oraciones alternativas \\
\hline Adjetivos prenominales & $53(23,04 \%)$ \\
\hline Adverbios en -mente & $19(8,26 \%)$ \\
\hline Construcciones pasivas & $111(48,26 \%)$ \\
\hline Per. “poder + infinitivo" & $47(20,43 \%)$ \\
\hline
\end{tabular}

Considerándose la cantidad de oraciones que se incluyeron en el cuestionario para cada recurso de interés, la tasa de sustitución de cada uno de estos resultó según se muestra en la Figura $2^{6}$ :

Figura 2. Tasas de sustitución de los recursos de interés

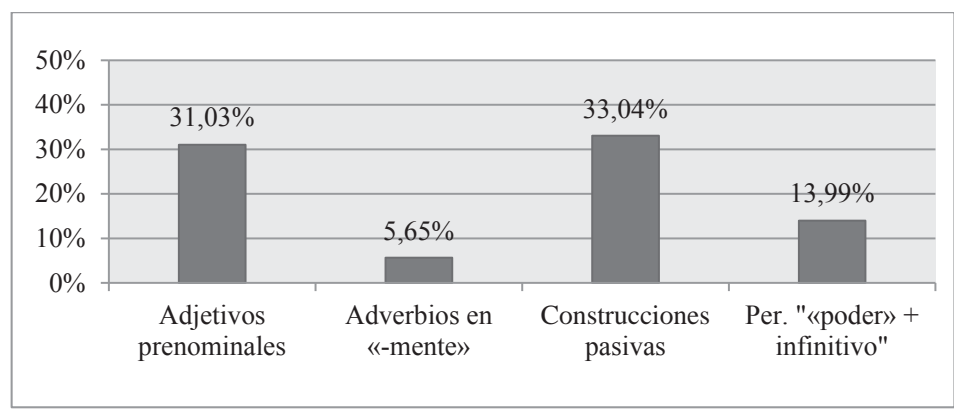

El recurso más sustituido, como se puede apreciar, corresponde a las construcciones pasivas con ser + participio, que son seguidas por los adjetivos prenominales, la perífrasis "poder + infinitivo" y, por último, los adverbios en -mente. Este resultado es coherente con las actitudes registradas, ya que se comprobó que mientras más baja fuera la media actitudinal de un recurso más sería este sustituido.

paréntesis, precedida del signo "menor que" $(<)$, la oración original a partir de la que surgió.

6 En este artículo se corrigieron las cifras correspondientes a las tasas de sustitución de los recursos de interés que aparecen en Echeverría 2015, las cuales, lamentablemente, eran incorrectas. De todos modos, la interpretación de estas cifras es la misma aquí y en dicha tesis, pues la ordenación fue idéntica en ambos casos a pesar del error de cálculo inicial. 


\subsubsection{Adjetivos prenominales}

En el caso de los adjetivos prenominales, se observaron cuatro alternativas en las respuestas de los informantes, distribuidas según muestra la Figura 3:

Figura 3. Alternativas frente al uso de adjetivos prenominales

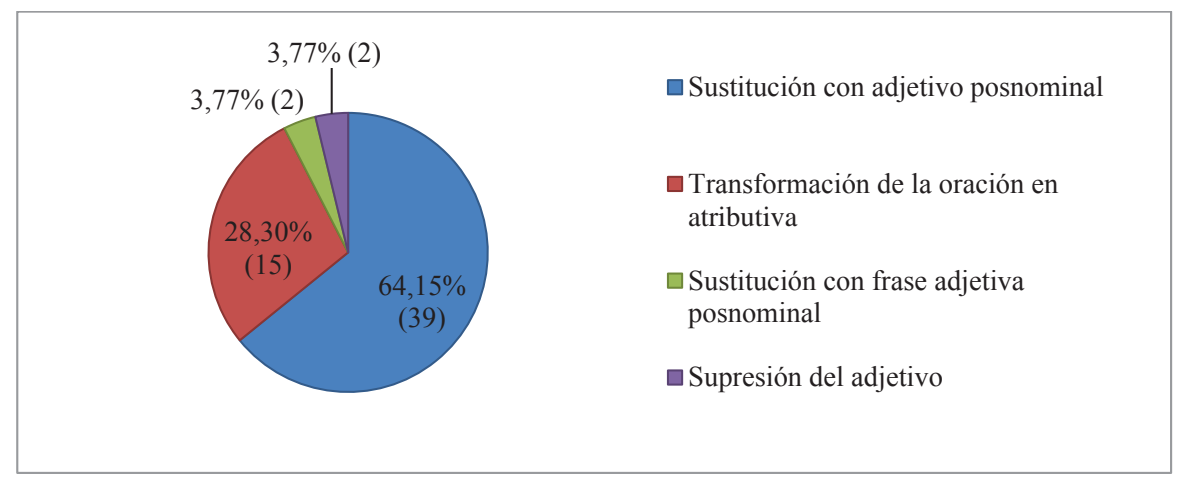

\subsubsection{Sustitución con adjetivo posnominal}

Esta alternativa consiste el uso de un adjetivo pospuesto al sustantivo sobre el que actúa. En la mayoría de los casos el adjetivo usado fue el mismo de la oración original, pero también se registraron casos en los que se optó por uno distinto. P. ej.: Ayer fue un día hermoso (< Ayer hubo un hermoso día), Incluso si adoptáramos su metodología se lograrían resultados iguales (< Se lograrían iguales resultados incluso si adoptáramos su metodología), Las nubes negras anunciaban el temporal que venía (< Las negras nubes anunciaban el temporal que venía).

\subsubsection{Transformación de la oración en atributiva}

Esta alternativa consiste en la transformación de la oración original en una oración atributiva, empleándose como atributo en todos los casos salvo en uno el mismo adjetivo utilizado originalmente. P. ej.: Ayer estuvo hermoso el día (< Ayer hubo un hermoso día), Ayer el día estuvo maravilloso (< ídem), Distinta era la situación que se vivía en los años setenta (<Distinta situación era la que se vivía en los años setenta).

\subsubsection{Sustitución con frase adjetiva posnominal}

Esta alternativa consiste el uso de una frase adjetiva libre (es decir, no de una unidad fraseológica) pospuesta al sustantivo sobre el que actúa: Mi padre era un abogado de estatura alta (< Mi padre era un alto abogado), Mi padre era un abogado muy importante (<ídem). Nótese que las dos oraciones alternativas proporcionadas muestran distintas interpretaciones del uso del adjetivo alto: mientras que en un 
caso este se interpreta como adverbial en relación con el ser abogado, en el otro se interpreta como un simple epíteto referente a la estatura.

\subsubsection{Supresión del adjetivo}

Esta alternativa consiste en la mera supresión del adjetivo en la frase nominal, sin ningún tipo de compensación para reexpresar su contenido: El candidato se reunirá con el partido esta semana (< El futuro candidato se reunirá con el partido esta semana), Será muy difícil encargarse de las denuncias (< Será muy difícil hacerse cargo de las numerosas denuncias).

\subsubsection{Adverbios en -mente}

En el caso de los adverbios en -mente las alternativas observadas fueron seis, distribuidas según muestra la Figura 4:

Figura 4. Alternativas frente al uso de adverbios en -mente

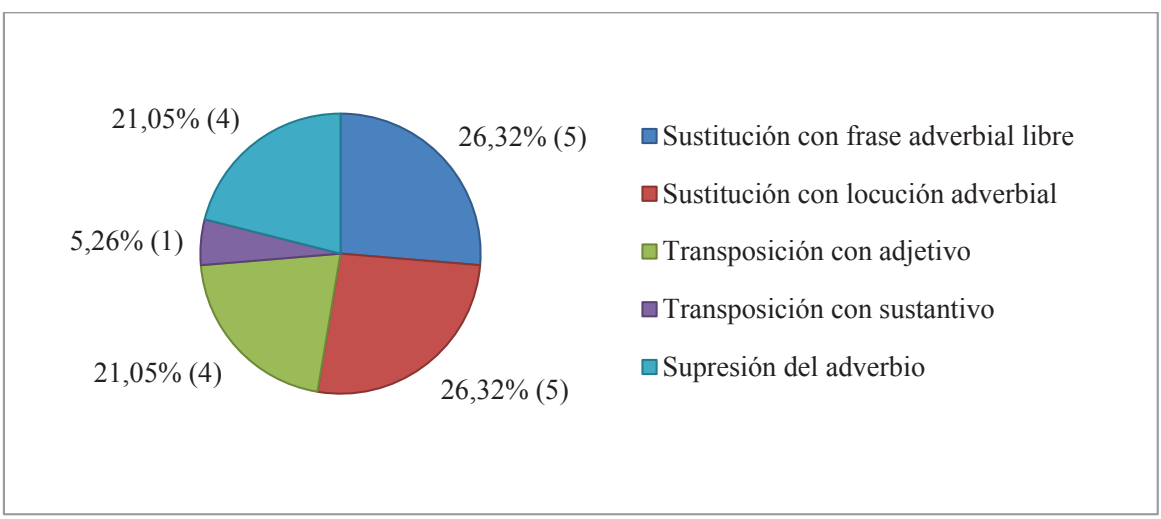

\subsubsection{Sustitución con frase adverbial libre}

Esta alternativa consiste en la sustitución del adverbio original con una frase adverbial libre, empleándose en cada caso la misma base léxica de aquel. P. ej.: El detenido había golpeado con violencia a su pareja (< El detenido había golpeado violentamente a su pareja), Fernando se volteó con brusquedad al oír su voz (< Fernando se volteó bruscamente al oír su voz), Se puso de pie con rapidez cuando el profesor lo llamó (< Se puso de pie rápidamente cuando el profesor lo llamó).

\subsubsection{Sustitución con locución adverbial}

Esta alternativa consiste en la sustitución del adverbio original con una locución (es decir, con una unidad fraseológica) adverbial, también en este caso empleándose 
siempre la misma base léxica de aquel. P. ej.: Si sigue así, de seguro reprobará la asignatura (< Seguramente reprobará la asignatura si sigue así), Esa posibilidad no se contempla en absoluto (< Esa posibilidad está absolutamente fuera de alcance).

\subsubsection{Transposición ${ }^{7}$ con adjetivo}

Esta alternativa consiste en la reexpresión del contenido del adverbio original mediante un adjetivo con la misma base léxica, en el sujeto o en el atributo de una oración atributiva, que en uno de los casos constatados corresponde a una oración de relativo: Si sigue así, lo más probable es que repruebe la asignatura (< Seguramente reprobará la asignatura si sigue así), Si sigue así, es muy probable que repruebe la asignatura ( $<$ ídem), Para que lo consideremos, debe entregar un proyecto cuya rigurosidad sea aceptable (<Debe entregar un proyecto suficientemente riguroso para que lo consideremos).

\subsubsection{Transposición con sustantivo}

Esta alternativa consiste en la reexpresión del contenido del adverbio original mediante un sustantivo, que en el único caso registrado corresponde al objeto directo de la oración: En aquella oración hice todo errado ( $<$ Aquella vez hice las cosas completamente mal).

\subsubsection{Supresión del adverbio}

Esta alternativa consiste en la mera supresión del adverbio original, sin ningún tipo de sustitución ni transposición para reexpresar su contenido. P. ej.: Debe entregar un proyecto riguroso para que lo consideremos ( $<$ Debe entregar un proyecto suficientemente riguroso para que lo consideremos), La exposición a la que asistí fue brillante (< La exposición a la que asistí fue totalmente brillante), Si sigue así reprobará la asignatura (< Seguramente reprobará la asignatura si sigue así).

\subsubsection{Construcciones pasivas con ser + participio}

En el caso de las construcciones pasivas con ser + participio las alternativas observadas fueron tres, distribuidas según muestra la Figura 5:

Cuando hablamos de transposición en este contexto nos referimos, en la línea de Vinay y Darbelnet (1995), a la reexpresión de la sustancia semántica de una unidad lingüística determinada mediante una unidad perteneciente a una categoría verbal distinta. La única diferencia es que dichos autores hablan de transposiciones interidiomáticas (francés-inglés), mientras que aquí hablamos de transposiciones intraidiomáticas (dentro del español). 
Figura 5. Alternativas frente al uso de construcciones pasivas con ser + participio

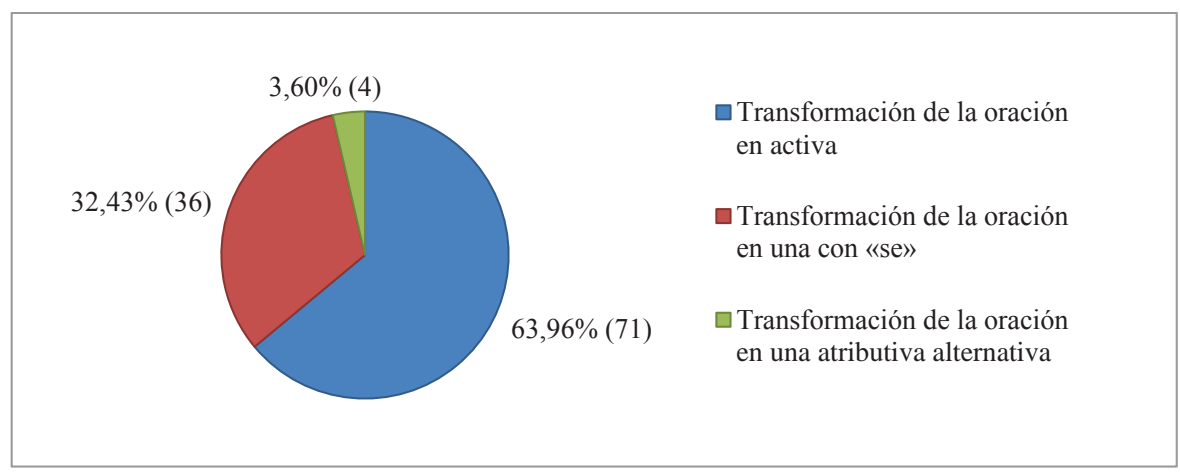

\subsubsection{Transformación de la oración en activa}

Esta alternativa consiste en la transformación de la oración original en una oración activa, es decir, con el sujeto como agente y el objeto directo como paciente. De las oraciones registradas, solo en dos de ellas el paciente se mantuvo antepuesto al agente, siendo lo común lo inverso (existiendo agente y paciente explícitos, claro). P. ej.: Uno de los organizadores del evento anunciará al ganador $(<$ El ganador será anunciado por uno de los organizadores del evento), A la niña de la esquina la asesinó algún loco cerca de su casa (< La niña de la esquina fue asesinada por algún loco cerca de su casa), La Cámara de Diputados aprobó el proyecto de ley (< El proyecto de ley fue aprobado por la Cámara de Diputados).

\subsubsection{Transformación de la oración en una con se}

Esta alternativa consiste en la transformación de la oración original en una oración construida con el pronombre se. P. ej.: Los resultados se publicarán durante la mañana ( $<$ Los resultados serán publicados durante la mañana), Se culpará a un inocente si no aparece el verdadero responsable (< Alguien inocente será culpado si el verdadero responsable no aparece), Según nuestras fuentes, se ha visto a alguien en la escena del crimen (< Según nuestras fuentes, alguien había sido visto en la escena del crimen).

\subsubsection{Transformación de la oración en una atributiva alternativa}

Esta alternativa consiste en la transformación de la oración original en una oración atributiva pasiva construida no con participio, sino con un sustantivo de significado léxico pasivo como núcleo de la frase que constituye el atributo, y en algunos casos con un verbo distinto de $\operatorname{ser}^{8}$. P. ej.: En la actualidad, cualquier persona puede ser blanco de un delincuente ( $<$ Hoy en día, cualquier persona puede ser atacada por un

\footnotetext{
Estas oraciones, en estricto rigor, son idénticas a las construidas con ser + participio en lo que a su pasividad respecta, independientemente de que estén construidas de manera diversa y de que por lo general no reciban el nombre de pasivas en la literatura especializada (Alarcos 1969).
} 
delincuente),Hoyendía,cualquierpersonapuedeservíctimadeundelincuente(<ídem), En estos tiempos, cualquiera puede ser víctima de la delincuencia (<ídem).

\subsubsection{Perífrasis "poder + infinitivo"}

En el caso de la perífrasis "poder + infinitivo" se observaron tres alternativas, distribuidas según muestra la Figura 6:

Figura 6. Alternativas frente al uso de la perífrasis "poder + infinitivo"

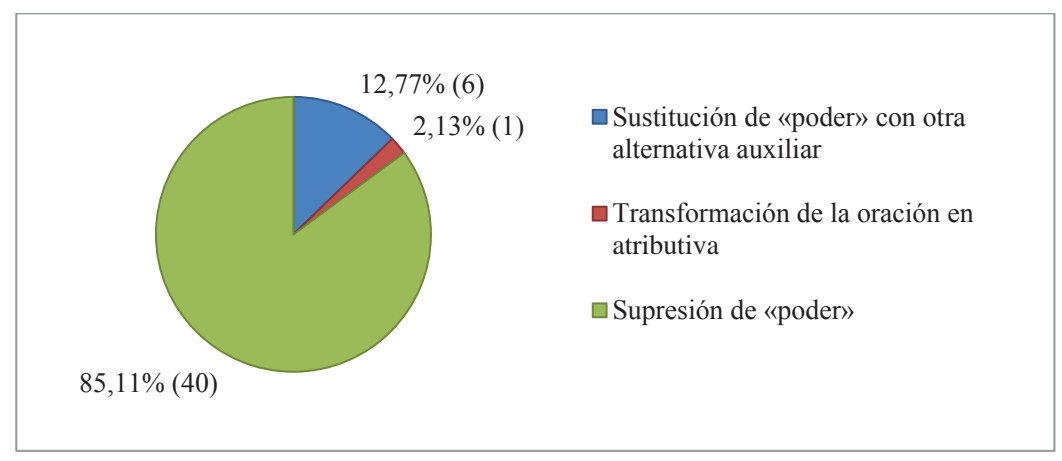

\subsubsection{Sustitución de poder con otra alternativa auxiliar}

Esta alternativa consiste en el uso de un verbo auxiliar alternativo o bien de una alternativa auxiliar pluriverbal construida con un sustantivo o adjetivo con significado léxico de posibilidad y la preposición de. P. ej.: Después de mucho tiempo la municipalidad, al fin, logró construir el puente (< Recién ahora la municipalidad pudo construir el puente), Si se me entrega un mapa seré capaz de llegar al evento sin dificultad (< Si me dan un mapa podré llegar al evento sin problemas), Quisiera tener la posibilidad de vender libros en la universidad (< Me gustaría poder vender libros en la universidad).

\subsubsection{Transposición con adjetivo}

Esta alternativa consiste en la reexpresión del contenido de la perífrasis mediante un adjetivo. En el único caso registrado, en que el informante optó por una oración atributiva, el adjetivo, que corresponde al atributo, se formó con una base léxica distinta de la del verbo auxiliado original y con el sufijo -ble, que significa posibilidad pasiva: Desde que me traicionó, esa persona ya no es confiable (< Ya no puedo creer en esa persona desde su última traición).

\subsubsection{Supresión de poder}

Esta alternativa consiste en la mera supresión poder, con el verbo en infinitivo o bien con la adopción del tiempo verbal correspondiente según el caso (sea con la 
mantención del mismo verbo, en cuanto unidad léxica, o con la adopción de otro), sin ningún tipo de sustitución ni transposición para reexpresar el contenido del verbo auxiliar. P. ej.: Para manejar un automóvil es necesario obtener el permiso legal (< Para poder manejar un automóvil es necesario obtener el permiso legal), Concéntrate y encontrarás las llaves (< Concéntrate y podrás encontrar las llaves), Si se me entrega un plano con indicaciones de cómo llegar al evento, lo haré sin problemas (< Si me dan un mapa podré llegar al evento sin problemas).

\section{DiSCUSIÓN Y CONCLUSIONES}

En cuanto a las actitudes registradas, al mostrar estas una tendencia marcadamente favorable, no parece descabellado conjeturar que el tratamiento de distanciamiento que en ocasiones reciben los recursos asociados a anglicismos de frecuencia no alcanza a tener un efecto importante en su percepción por parte de los aprendices de traductor, al menos en ausencia de mayores estímulos metalingüísticos, lo cual sería coherente con la proliferación de dichos recursos en la traducción desde el inglés. Sin embargo, por supuesto, tal conjetura solo podría verse confirmada (o desestimada) con la realización de nuevas investigaciones.

Un hecho interesante que podría servir para matizar lo anterior es que las construcciones pasivas con ser + participio, a pesar de haber contado con una media que se halla en el segmento de la favorabilidad $(3,71)$, correspondieron al recurso de interés peor evaluado (y el único con una media menor que 4$)^{9}$. La razón por la que consideramos esto interesante es que estas construcciones parecen ser el recurso sobre cuya sobreutilización anglicante más se ha advertido en la literatura (Real Academia Española 1973; Vázquez-Ayora 1977; Pratt 1980; Calvo 1983; García Yebra 1984; Aguado 1990; Arauz 1992; Lorenzo 1991, 1996; Alcaraz y Martínez 1997; López y Minett 1997; García González 1997-1998; Rodríguez 2000, 2002; Montes 2003; Fernández 2008), destacándose entre sus denunciantes la Real Academia Española (1973: 451), máximo símbolo de autoridad en lo que respecta al español, que en su Esbozo escribía: "Las lenguas francesa e inglesa emplean la pasiva [...] en proporciones mucho mayores que la nuestra. Conviene que los traductores tengan en cuenta esta preferencia, para no cometer faltas de estilo y aun incorrecciones gramaticales" (cfr. Kock y Gómez 1985). Por esto, aun si las actitudes registradas tendieron a ser favorables, no deja de ser pertinente volver a abogar en este punto por un tratamiento adecuado de temas como el del anglicismo de frecuencia en la formación de traductores y en el estudio del contacto interlingüístico en general, temas que, como se indicó en la introducción, no han estado exentos de confusiones.

Por lo que respecta a las preferencias alternativas de los informantes, las cuales fueron coherentes con las actitudes registradas, este estudio constituye un aporte a la formación de traductores inglés-español (y eventualmente de hablantes de español en general), pues viene a complementar, desde una perspectiva descriptiva, los trabajos anteriores que se han ocupado de proporcionar alternativas a los recursos de interés, trabajos que por lo general han adoptado una óptica más bien normativa.

Curiosamente, esto se opone diametralmente a lo observado en el estudio piloto, donde dicho recurso fue el mejor evaluado En nuestra interpretación, lo más probable es que los resultados dispares de ambas indagaciones en relación con las construcciones pasivas con ser + participio se debieran a variables de los establecimientos educacionales y de los programas de traducción, tales como el currículo y la formación de los docentes. 
En cuanto a la diversidad de las alternativas registradas, se constató la existencia de fórmulas de sustitución relativamente estables, aunque de todos modos no creemos que tal estabilidad refleje, en general, una limitación importante de los informantes por lo que respecta a su creatividad sustitutiva. En este sentido, debe tenerse en cuenta que la organización de las preferencias se hizo mediante una serie de abstracciones metodológicas, para dar cierta sistematicidad los datos obtenidos, y que de todas formas hubo variedad dentro de buena parte de los grupos establecidos.

Por otra parte, resulta destacable que, salvo en el caso de la perífrasis "poder + infinitivo", fueron pocos los casos de mera supresión que se registraron, lo que sugiere que los informantes tendieron a intentar reflejar de algún modo los recursos de interés en la producción de sus oraciones alternativas, sin omitir el contenido atribuido a las oraciones originales, conducta que resulta valorable en futuros traductores, quienes deben ser capaces de lograr niveles altos de invariación semántica en el desempeño de su labor. En cuanto al caso de la perífrasis "poder + infinitivo", la alta cantidad de supresiones de poder resultaba en cierto modo esperable, ya que con frecuencia se ha dicho, en especial al contrastar el español con el inglés, que el uso de este verbo auxiliar, que expresa posibilidad (siendo la posibilidad prerrequisito lógico de la acción), en muchos casos resulta superfluo (Vázquez-Ayora 1977; Lorenzo 1996; Rodríguez 2000, 2002).

Para concluir, este estudio podría ser complementado por nuevas indagaciones que aborden el tema de los recursos asociados a anglicismos de frecuencia hispánicos o a fenómenos de interferencia de frecuencia en general desde distintas perspectivas. P. ej., sería interesante una investigación en que se considerasen las creencias, las actitudes y las preferencias no solo de estudiantes, sino también de profesores de traducción; o también se podrían comparar las actitudes y las alternativas aquí registradas con las de estudiantes de inglés y de español no pertenecientes a programas de traducción, para determinar si el currículo tiene alguna incidencia a este respecto. De este modo, se podrá seguir contribuyendo a la comprensión de los contextos en que se produce el contacto interlingüístico.

\section{OBRAS CITADAS}

Aguado de Cea, Guadalupe. 1990. "Interferencias lingüísticas en los textos técnicos”. En II Encuentros Complutenses en Torno a la Traducción, 12-16 de diciembre de 1988. Madrid: Instituto Universitario de Lenguas Modernas y Traductores, Universidad Complutense. 163-169.

Alarcos Llorach, Emilio. 1969. Gramática estructural según la escuela de Copenhague y con especial atención a la lengua española. Madrid: Gredos.

.1999. Gramática de la lengua española. Madrid: Espasa.

Alcaraz Varó, Enrique y María Antonia Martínez Linares. 1997. Diccionario de lingüística moderna. Barcelona: Ariel.

Arauz, Pedro. 1992. "Errores frecuentes en la traducción”. En Sebastián Barrueco, Lina Sierra y María José Sánchez (Eds.), Actas de las I Jornadas Internacionales de Inglés Académico, Técnico y Profesional: Investigación y Enseñanza. Alcalá de Henares: Servicio de Publicaciones de la Universidad de Alcalá de Henares. 179-183.

Bosque, Ignacio y Violeta Demonte (Dirs.). 1999. Gramática descriptiva de la lengua española. Madrid: Espasa-Calpe. 
Calvo Montoro, María José. 1983. La voz pasiva. Madrid: Coloquio.

Cooper, Robert L. y Joshua A. Fishman. 1974. "The study of language attitudes". International Journal of the Sociology of Language 3: 5-20.

Coseriu, Eugenio. 1982. "Determinación y entorno: dos problemas de una lingüística del hablar". En Teoría del lenguaje y lingüística general: cinco estudios. Madrid: Gredos. 282323.

Demonte, Violeta. 1999. "El adjetivo: clases y usos. La posición del adjetivo en el sintagma nominal”. En Ignacio Bosque y Violeta Demonte (Dirs.), Gramática descriptiva de la lengua española. Madrid: Espasa-Calpe. 129-215.

Domínguez de Rodríguez-Pasqués, Petrona. 1970. "Morfología y sintaxis del adverbio en -mente". En Carlos H. Magis (Ed.), Actas del Tercer Congreso Internacional de Hispanistas. México: El Colegio de México. 293-303.

Eagly, Alice Hendrickson y Shelly Chaiken. 1993. The Psychology of Attitudes. Londres: Harcourt Brance College Publishers.

Echeverría Arriagada, Carlos I. 2011. "Sobre el uso de adverbios en -mente en la traducción inglés-castellano". Ponencia presentada en el XI Congreso Nacional de Estudiantes de Traducción e Interpretación (Valparaíso, 27 y 28 de octubre).

2014. "Las actitudes de estudiantes de traducción inglés-español hacia recursos gramaticales del español asociados a anglicismos de frecuencia”. Contextos: estudios de humanidades y ciencias sociales 31: 49-68.

2015. Actitudes y preferencias de estudiantes de traducción inglés-español frente a recursos gramaticales del español asociados a anglicismos de frecuencia. Tesis de magíster, Universidad de Santiago de Chile, Santiago.

.2016. "La interferencia lingüística de frecuencia". Boletín de filología LI (1): 93-115.

Fernández Marrero, Ana. 2008. "La sintaxis y la ortografía: dos niveles lingüísticos bajo la influencia del inglés". Revista de filología 26: 31-45.

García González, José Enrique. 1997-1998. "Anglicismos morfosintácticos en la traducción periodística (inglés-español): análisis y clasificación”. Cauce: revista de filología y su didáctica 20-21: 593-622.

García Yebra, Valentín. 1984. Teoría y práctica de la traducción. Madrid: Gredos.

García-Page, Mario. 1991. "Breves apuntes sobre el adverbio en -mente". Thesaurus XLVI (2): $183-224$

Garrett, Peter. 2010. Attitudes to language. Cambridge: Cambridge University Press.

Gili Gaya, Samuel. 1980. Curso superior de sintaxis española. Barcelona: Vox.

Gómez Torrego, Leonardo. 2003. Ejercicios de gramática normativa II. Madrid: Arco Libros. Grijelmo, Álex. 1997. Defensa apasionada del idioma español. Madrid: Taurus.

Ivir, Vladimir. 1991. "Contrastive methods in contact linguistics". En Vladimir Ivir y Damir Kalogjera (Eds.), Languages in Contact and Contrast: Essays in Contact Linguistics. Nueva York: Mouton de Gruyter. 237-245.

Klein-Andreu, Flora. 1985. "La cuestión de anglicismo: apriorismo y métodos". Thesaurus XL: 533-548.

Kock, Josse de y Carmen Gómez Molina. 1985. "La frecuencia de la pasiva en español y otras lenguas". Revista española de lingüística 15 (1): 117-132.

Lázaro Carreter, Fernando. 1997. El dardo en la palabra. Barcelona: Galaxia Gutenberg.

López Guix, Juan Gabriel y Jacqueline Minett Wilkinson. 1997. Manual de traducción. Barcelona: Gedisa.

López Morales, Humberto. 1993. Sociolingüística. Madrid: Gredos.

Lorenzo, Emilio. 1980. "El anglicismo en la España de hoy". En El español de hoy, lengua en ebullición. Madrid: Gredos. 96-121.

1991. "Anglicismos y traducciones". Studia Patriciae Shaw Oblata II: 67-79. 1995-1996. "El anglicismo, problema hispánico”. Boletín de filología XXXV: 261-274. 
.1996. Anglicismos hispánicos. Madrid: Gredos.

Montes, José Joaquín. 2003. "La actual crisis de la voz pasiva en español”. Boletín de filología XXXIX: 103-121.

Moreno Fernández, Francisco. 2005. Principios de sociolingüística y sociología del lenguaje. Barcelona: Ariel.

Osgood, Charles E., George. J. Suci y Percy H. Tannenbaum. 1975. La medida del significado. Madrid: Gredos.

Pratt, Chris. 1980. El anglicismo en el español peninsular contemporáneo. Madrid: Gredos.

Rabanales, Ambrosio. 2005. "Queísmo y dequeísmo en el español de Chile”. Onomázein 12: 23-53.

Real Academia Española. 1973. Esbozo de una nueva gramática de la lengua española. Madrid: Espasa-Calpe 2005. Diccionario panhispánico de dudas. Madrid: Santillana.

Real Academia Española y Asociación de Academias de la Lengua Española. 2009. Nueva gramática de la lengua española. Madrid: Espasa.

. 2010. Nueva gramática de la lengua española: manual. Madrid: Espasa.

Rodríguez Medina, María Jesús. 2000. Los anglicismos de frecuencia sintácticos en los manuales de informática traducidos. Tesis doctoral, Universidad de Las Palmas de Gran Canaria, Las Palmas de Gran Canaria. Disponible en http://acceda.ulpgc.es/handle/1055 $3 / 2100$ ?nitem $=0 \&$ anterior $=\&$ siguiente $=$

. 2002. "Los anglicismos de frecuencia sintácticos en español". Revista española de lingüística aplicada 15: 149-170.

Thomason, Sarah Grey y Terrence Kaufman. 1988. Language Contact, Creolization, and Genetic Linguistics. Berkeley: University of California.

Thurstone, L. L. 1928. "Attitudes can be measured”. American Journal of Sociology 33: 529554.

Urbán Fernández, F. J. 1980. "Un método de investigación de origen psicolingüístico: el diferencial semántico". Cauce 3: 47-69.

Vázquez-Ayora, Gerardo. 1977. Introducción a la traductología: curso básico de traducción. Washington: Georgetown University Press.

Vendler, Zeno. 1967. Linguistics and Philosophy. Ithaca: Cornell University Press.

Vinay, Jean-Paul y Jean Darbelnet. 1995. Comparative Stylistics of French and English. Ámsterdam: John Benjamins. 
APÉndice: CONTENIDO DEL CUESTIONARIO ${ }^{10}$

1) Recién ahora la municipalidad pudo construir el puente.

No ACEPTABLE

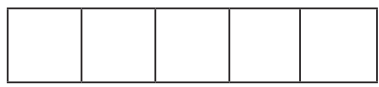

Aceptable

ORACIÓN ALTERNATIVA (EN CASO DE HABERLA):

2) Esa posibilidad está absolutamente fuera de alcance.

No ACEPTABLE

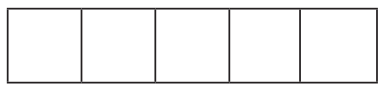

ACEPTABLE

ORACIÓN ALTERNATIVA (EN CASO DE HABERLA):

3) El gobernador decidió no referirse al tema.

No ACEPTABLE

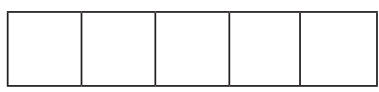

ACEPTABLE

ORACIÓN ALTERNATIVA (EN CASO DE HABERLA):

4) Pedro fue regañado por sus superiores después del evento.

No ACEPTABLE

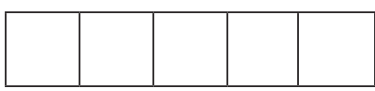

Aceptable

Oración ALTERNATIVA (EN CASO DE HABERLA):

5) Tiene que reconocer de que yo tenía razón.

No ACEPTABLE

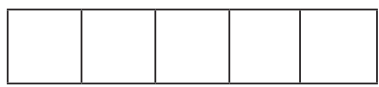

Aceptable

ORACiÓn ALTERNATIVA (EN CASO DE HABERLA):

10 Las oraciones de interés han sido resaltadas en negrita. 
6) Si me dan un mapa podré llegar al evento sin problemas.

No ACEPTABLE

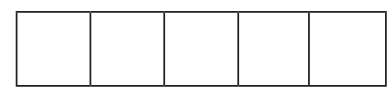

Aceptable

ORACiÓn ALTERNATIVA (EN CASO DE HABERLA):

7) El proyecto de ley fue aprobado por la Cámara de Diputados.

No ACEPTABLE

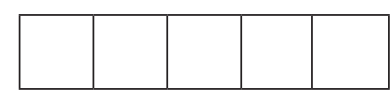

ACEPTABle

ORACIÓN ALTERNATIVA (EN CASO DE HABERLA):

8) El lunes le voy a enviarle los documentos sin falta.

No ACEPTABLE

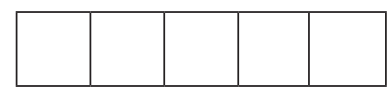

ACEPTABLe

ORACiÓN ALTERNATIVA (EN CASO DE HABERLA):

9) La niña de la esquina fue asesinada por algún loco cerca de su casa.

No ACEPTABle

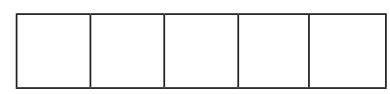

Aceptable

ORACIÓN ALTERNATIVA (EN CASO DE HABERLA):

10) La exposición a la que asistí fue totalmente brillante.

No ACEPTABLE

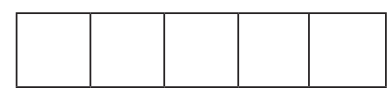

Aceptable

Oración ALTERnATIVA (EN CASO DE HABERLA): 
11) Nunca antes había tenido la oportunidad de asistir a un evento como este.

NO ACEPTABLE

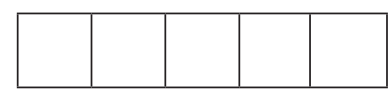

ACEPTABle

Oración ALternativa (en CASO DE haberla):

12) Todo el alumnado deberá presentarse a primera hora mañana.

No ACEPTABLE

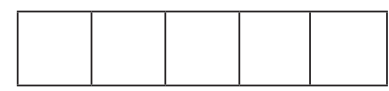

ACEPTABLE

ORACiÓN ALTERNATIVA (EN CASO DE HABERLA):

13) El ganador será anunciado por uno de los organizadores del evento.

No ACEPTABLE

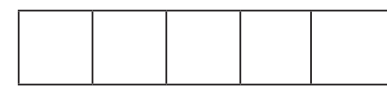

ACEPTABLE

Oración ALternativa (en CASO DE haberla):

14) Ya no puedo creer en esa persona desde su última traición.

No ACEPTABLE

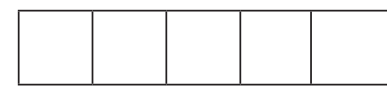

Aceptable

Oración ALTERnATIVA (EN CASO DE HABERLA):

15) Salga afuera de inmediato, por favor.

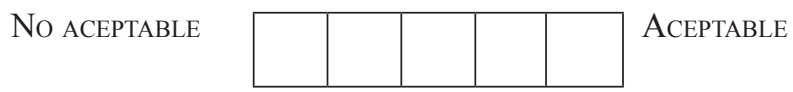

ORACIÓN ALTERNATIVA (EN CASO DE HABERLA): 
16) Según nuestras fuentes, alguien había sido visto en la escena del crimen.

No ACEPTABLE

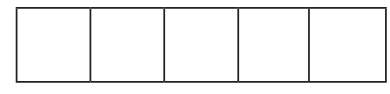

Aceptable

ORACiÓn ALTERNATIVA (EN CASO DE HABERLA):

17) Hoy en día, cualquier persona puede ser atacada por un delincuente.

No ACEPTABLE

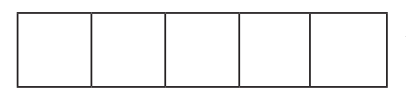

ACEPTABle

Oración Alternativa (EN CASO DE haberla):

18) Mi padre me decía de que nunca debía dejarme insultar.

No ACEPTABLE

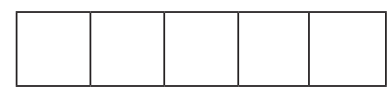

Aceptable

ORACIÓN ALTERNATIVA (EN CASO DE HABERLA):

19) Alguien de nosotros podría estar siendo observado por el asesino en este instante

No ACEPTABLE

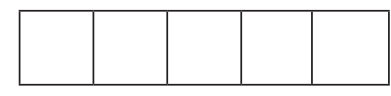

Aceptable

ORACiÓN ALTERNATIVA (EN CASO DE HABERLA):

20) Si entro a la universidad podré saber todo lo que me interesa.

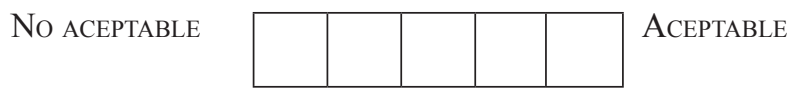

Oración ALTERnATIVA (EN CASO DE HABERLA): 


\section{1) Toda persona es observada por Dios}

No ACEPTABLE

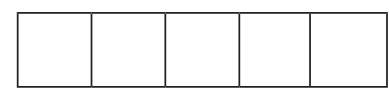

ACEPTABLE

ORACiÓN ALTERNATIVA (EN CASO DE HABERLA):

\section{2) Otro candidato será elegido por nosotros en su lugar}

No ACEPTABLE

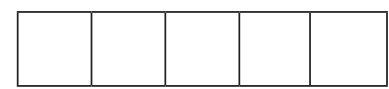

Aceptable

ORACión ALTERnATIVA (EN CASO DE HABERLA):

23) El futuro candidato se reunirá con el partido esta semana.

No ACEPTABLE

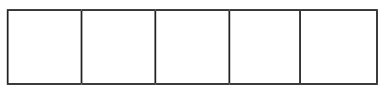

Aceptable

ORACiÓN ALTERNATIVA (EN CASO DE HABERLA):

\section{4) Ayer hubo un hermoso día.}

No ACEPTABLe

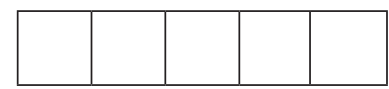

ACEPTABLe

ORACIÓN ALTERNATIVA (EN CASO DE HABERLA):

25) El detenido había golpeado violentamente a su pareja.

No ACEPTABLE

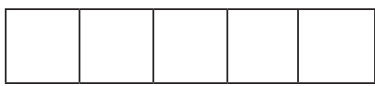

ACEPTABLE

ORACIÓN ALTERNATIVA (EN CASO DE HABERLA): 
26) No hay nada más hermoso que los verdes pastizales del sur.

No ACEPTABLE

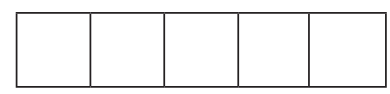

ACEPTABLe

Oración ALTERnativa (EN CASO DE haberla):

27) Distinta situación era la que se vivía en los años setenta.

No ACEPTABLE

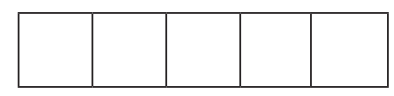

ACEPTABle

Oración Alternativa (EN CASO DE haberla):

28) Se lograrían iguales resultados incluso si adoptáramos su metodología.

No ACEPTABLE

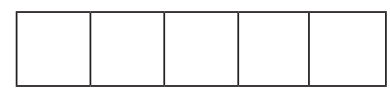

Aceptable

ORACIÓN ALTERNATIVA (EN CASO DE HABERLA):

29) Fernando se volteó bruscamente al oír su voz.

No ACEPTABLE

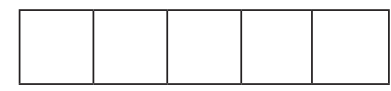

ACEPTABLE

ORACIÓN ALTERNATIVA (EN CASO DE HABERLA):

30) Será muy difícil hacerse cargo de las numerosas denuncias.

No ACEPTABLE

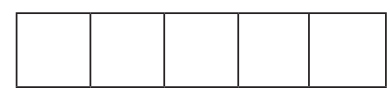

Aceptable

Oración ALTERnATIVA (EN CASO DE HABERLA): 
31) Las negras nubes anunciaban el temporal que venía.

No ACEPTABLE

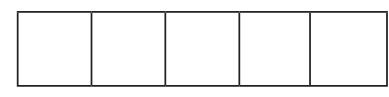

ACEPTABLE

ORACIÓN ALTERNATIVA (EN CASO DE HABERLA):

32) Debe entregar un proyecto suficientemente riguroso para que lo consideremos.

No ACEPTABLE

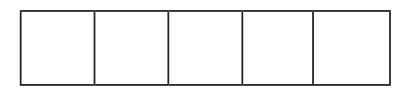

ACEPTABLE

ORACiÓN ALTERNATIVA (EN CASO DE HABERLA):

33) Algún día se lo tengo que decírselo.

No ACEPTABLE

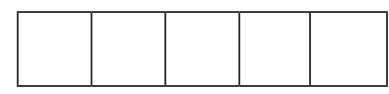

ACEPTABle

Oración ALternativa (en CASO DE haberla):

34) Desgraciadamente, no había nada más que se pudiera hacer.

No ACEPTABLE

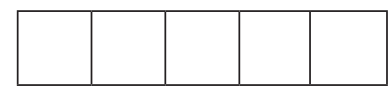

Aceptable

ORACIÓN ALTERNATIVA (EN CASO DE HABERLA):

35) Para poder manejar un automóvil es necesario obtener el permiso legal.

No ACEPTABLE

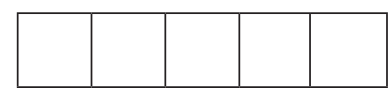

ACEPTABle

ORACIÓN ALTERNATIVA (EN CASO DE HABERLA): 
36) Mi sueño es poder trabajar en el extranjero.

No ACEPTABLE

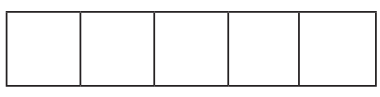

ACEPTABLE

Oración ALTERnativa (EN CASO DE HABERLa):

37) Esta hierba tiene propiedades sumamente beneficiosas para la salud.

No ACEPTABLE

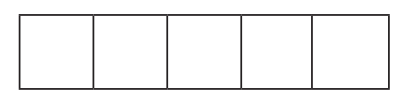

ACEPTABLE

ORACIÓN ALTERNATIVA (EN CASO DE HABERLA):

38) Me gustaría poder vender libros en la universidad.

No ACEPTABLe

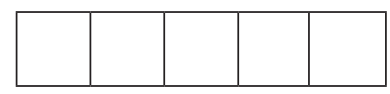

ACEPTABLe

ORACIÓN ALTERNATIVA (EN CASO DE HABERLA):

39) Mi hijo fue muy bien evaluado por sus profesores.

No ACEPTABle

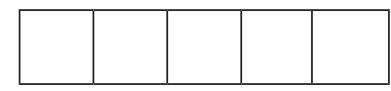

Aceptable

ORACIÓN ALTERNATIVA (EN CASO DE HABERLA):

40) Aquella vez hice las cosas completamente mal.

No ACEPTABLE

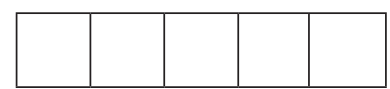

AcEPTABLe

Oración ALTERnATIVA (EN CASO DE HABERLA): 
41) Definitivamente se trata de una alternativa que hay que tener en cuenta.

No ACEPTABLE

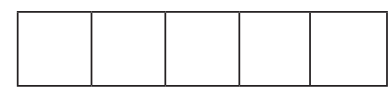

ACEPTABLE

ORACIÓN ALTERNATIVA (EN CASO DE HABERLA):

42) Afuera del colegio hay un ciego que no ve.

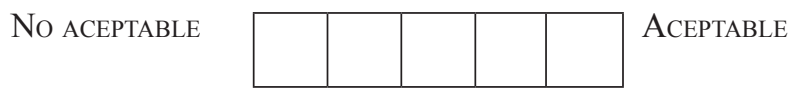

ORACiÓN ALTERNATIVA (EN CASO DE HABERLA):

43) Me preparé durante tres años con el fin de poder alcanzar la cima.

No ACEPTABLE

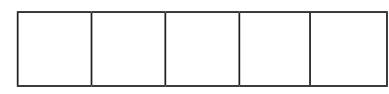

ACEPTABle

Oración ALternativa (en CASO DE haberla):

44) Todos los presentes votaron a favor de la propuesta.

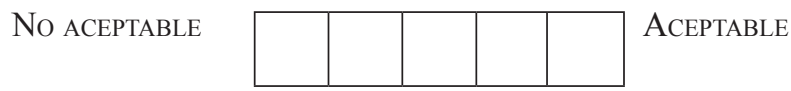

ORACIÓN ALTERNATIVA (EN CASO DE HABERLA):

45) Seguramente reprobará la asignatura si sigue así.

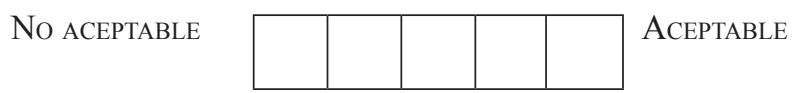

ORACIÓN ALTERNATIVA (EN CASO DE HABERLA): 
46) Aquel día al fin pude leer el diario.

No ACEPTABLE

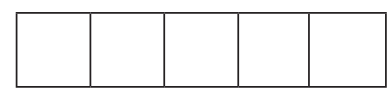

ACEPTABLe

Oración ALTERnATIVA (EN CASO DE HABERLA):

47) Mañana podré comer un plato de mi comida favorita.

No ACEPTABLE

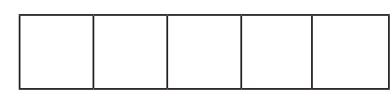

ACEPTABLe

ORACiÓN ALTERNATIVA (EN CASO DE HABERLA):

48) Con esfuerzo podré tener todo el dinero que quiero.

No ACEPTABLe

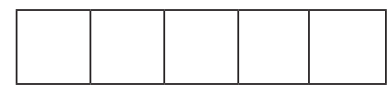

ACEPTABLE

ORACIÓN ALTERNATIVA (EN CASO DE HABERLA):

49) Al oír mi respuesta, me señaló de que estaba equivocado.

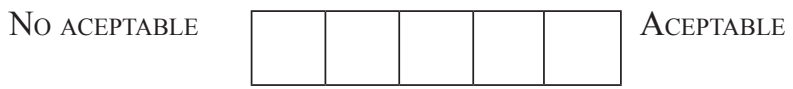

ORACIÓN ALTERNATIVA (EN CASO DE HABERLA):

50) Concéntrate y podrás encontrar las llaves.

No ACEPTABLE

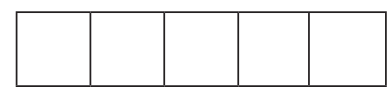

Aceptable

Oración alternativa (EN CASO DE HABERLa): 
51) Tengo entendido que la secretaria va a subir para arriba ahora.

No ACEPTABLE

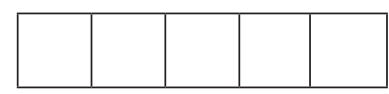

ACEPTABle

Oración ALTERnATiva (EN CASO DE HABERLa):

52) Alguien inocente será culpado si el verdadero responsable no aparece.

No ACEPTABLE

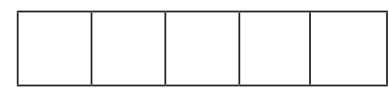

ACEPTABle

ORACiÓn ALTERnATIVA (EN CASO DE HABERLA):

53) Los resultados serán publicados durante la mañana.

No ACEPTABLE

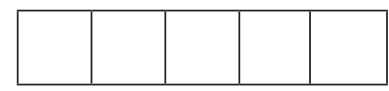

ACEPTABLE

Oración ALternativa (en CASO DE haberla):

54) Estoy completamente de acuerdo.

No ACEPTABLE

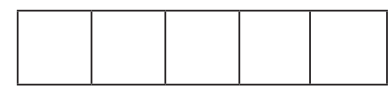

ACEPTABLE

Oración ALTERnATIVA (EN CASO DE HABERLA):

55) El señor Morales dijo que se iba a irse temprano.

No ACEPTABLE

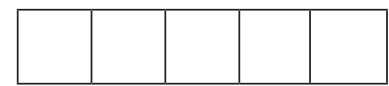

ACEPTABLe

ORACIÓN ALTERNATIVA (EN CASO DE HABERLA): 
56) La persona que quiero presentarle es un viejo amigo.

No ACEPTABLE

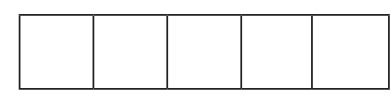

Aceptable

Oración ALternativa (EN CASO DE HABERLA):

57) Mi padre era un alto abogado.

No ACEPTABLE

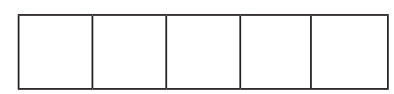

Aceptable

ORACIÓN ALTERNATIVA (EN CASO DE HABERLA):

58) Se puso de pie rápidamente cuando el profesor lo llamó.

No ACEPTABLE

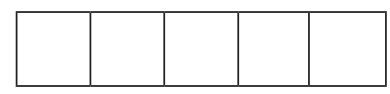

Aceptable

ORACIÓN ALTERNATIVA (EN CASO DE HABERLA): 